\title{
Usefulness of 2D-Perfusion Analysis for Original the Assessment of Unilateral Cervical Internal Carotid Artery Stenosis
}

Akifumi Yoshikawa, Takehiro Uno, Iku Nambu, Tomoya Kamide, Kouichi Misaki, and Mitsutoshi Nakada

Objective: We investigated the usefulness of 2D-perfusion analysis for the evaluation of cerebral blood flow in unilateral cervical internal carotid artery stenosis.

Methods: We conducted a 2D-perfusion analysis during cerebral angiography and ${ }^{123}$-iodoamphetamine (IMP) single photon emission computed tomography (SPECT) for unilateral cervical internal carotid artery stenosis without contralateral stenosis. The relationship between the ratio of the lesion side to the normal side in the parameters obtained by 2D-perfusion and the value calculated by stereotactic extraction estimation (SEE) analysis of SPECT was statistically examined.

Results: The ratios of the lesion side to the normal side regarding the peak arrival time (AT; time to peak [TTP]) of the contrast agent and the mean filling time (mean transit time [MTT]) of the contrast agent in 2D-perfusion significantly correlated with the area of Stage II and increase ratio (I.R) $\leq 30 \%$ in the SEE analysis $(p=0.002,0.003)$.

Conclusion: 2D-perfusion analysis can be used to estimate the extent of impaired cerebrovascular reserve (CVR) area in unilateral internal carotid artery stenosis.

Keywords $>$ internal carotid artery stenosis, 2D-perfusion, digital subtraction angiography, single photon emission computed tomography, cerebrovascular reserve

\section{Introduction}

In patients with cervical internal carotid artery stenosis, it is important to evaluate the instability of plaques and state of cerebral blood flow to predict ischemic stroke/hyperperfusion after revascularization and treatment indication. ${ }^{1-6)}$ To assess cerebral blood flow, positron emission tomography (PET) and acetazolamide (ACZ)-loaded single photon emission computed tomography (SPECT) are used in many cases. However, these procedures cannot be performed at some institutions. Furthermore, the use of ACZ for SPECT is not covered by health insurance in Japan.

Department of Neurosurgery, University of Kanazawa, Kanazawa, Ishikawa, Japan

Received: June 30, 2020; Accepted: November 30, 2020

Corresponding author: Akifumi Yoshikawa. Department of Neurosurgery, University of Kanazawa, 13-1, Takaramachi, Kanazawa, Ishikawa 920-8641, Japan

Email: yoshikawa@med.kanazawa-u.ac.jp

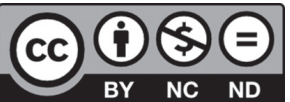

This work is licensed under a Creative Commons Attribution-NonCommercial-

NoDerivatives International License.

(C)2021 The Japanese Society for Neuroendovascular Therapy
Serious adverse reactions have been reported ${ }^{7,8)}$; therefore, it was recommended that the cerebrovascular reserve (CVR) be predicted using other methods. ${ }^{9)}$

Application software (2D-perfusion: AlluraClarity FD20/15 and Interventional Workspot, Philips Healthcare, Best, Netherlands) facilitates the assessment of cerebral blood flow based on cerebral angiographic images. However, only a few previous studies reported its usefulness ${ }^{10-12)}$; no evaluation method has been established.

In this study, we investigated the correlation between 2D-perfusion and ACZ-loaded SPECT in patients with unilateral cervical internal carotid artery stenosis.

\section{Materials and Methods}

The protocol of this study was approved by the ethics review board of our institution. Informed consent regarding cerebral angiography and ACZ usage was received from all patients.

\section{Patients}

The subjects were 28 patients with cervical internal carotid artery stenosis on whom 2D-perfusion on cerebral angiography and ACZ-loaded SPECT with ${ }^{123}$ I-iodoamphetamine 
(IMP) were performed around the same time at our institution between November 2016 and April 2019. Of these, 14 were analyzed, excluding 3 in whom 2D-perfusion or SPECT was insufficient for analysis and 11 who did not meet the definition of the normal side, which was being free from $\geq 50 \%$ stenosis of intra-/extracranial blood vessels involving the cervical internal carotid artery to middle cerebral artery (MCA) on the North American Symptomatic Carotid Endarterectomy Trial (NASCET) or Warfarin Aspirin Symptomatic Intracranial Disease (WASID) methods.

\section{D-perfusion (Fig. 1)}

Using an anteroposterior view obtained by inserting a $4 \mathrm{Fr}$ pigtail catheter (Cook Medical, Bloomington, IN, USA) into the ascending aorta and infusing $20 \mathrm{~mL}$ of contrast medium at a rate of $10 \mathrm{~mL} / \mathrm{second}$ on cerebral angiography through femoral or brachial artery puncture, 2D-perfusion analysis was conducted. On the image, a median line was established and the symmetric regions of interest were established in the bilateral MCA areas to prepare a time-concentration curve (Fig. 1A). The arrival time (AT) of contrast medium, time to peak (TTP), wash-in rate (WiR), retention time of contrast medium (Width), area under the curve (AUC), and mean transit time (MTT) for the bilateral regions of interest were automatically calculated from the curve (Fig. 1B). In this study, we adopted the lesion-side-to-normal-side ratio of each parameter, and abbreviated the parameters as rAT, rTTP, rWiR, rWidth, rAUC, and rMTT, respectively.

\section{ACZ-loaded SPECT with 123I-IMP}

We used the dual table ARG method in which resting and ACZ-loaded SPECT is conducted during a single examination session through 1-point arterial blood collection. Stereotactic extraction estimation (SEE) analysis ${ }^{13)}$ was performed, and the rates of stage II, with a marked reduction in CVR in the MCA area, and an area with an increase ratio (I.R) of $\leq 30 \%$ were calculated. Furthermore, CVR in the entire MCA area was used.

\section{Statistical analysis}

Based on the values obtained on 2D-perfusion and SPECT, Spearman's correlation coefficient ( $\mathrm{r}$ ) was calculated. A value of $\geq 0.70$ or $\leq 0.70$ was regarded as demonstrating a strong correlation. A p value of 0.05 was regarded as significant.

\section{Results}

The registered patients consisted of 12 males and 2 females, with a mean age of 72 years (56-87 years). The right side
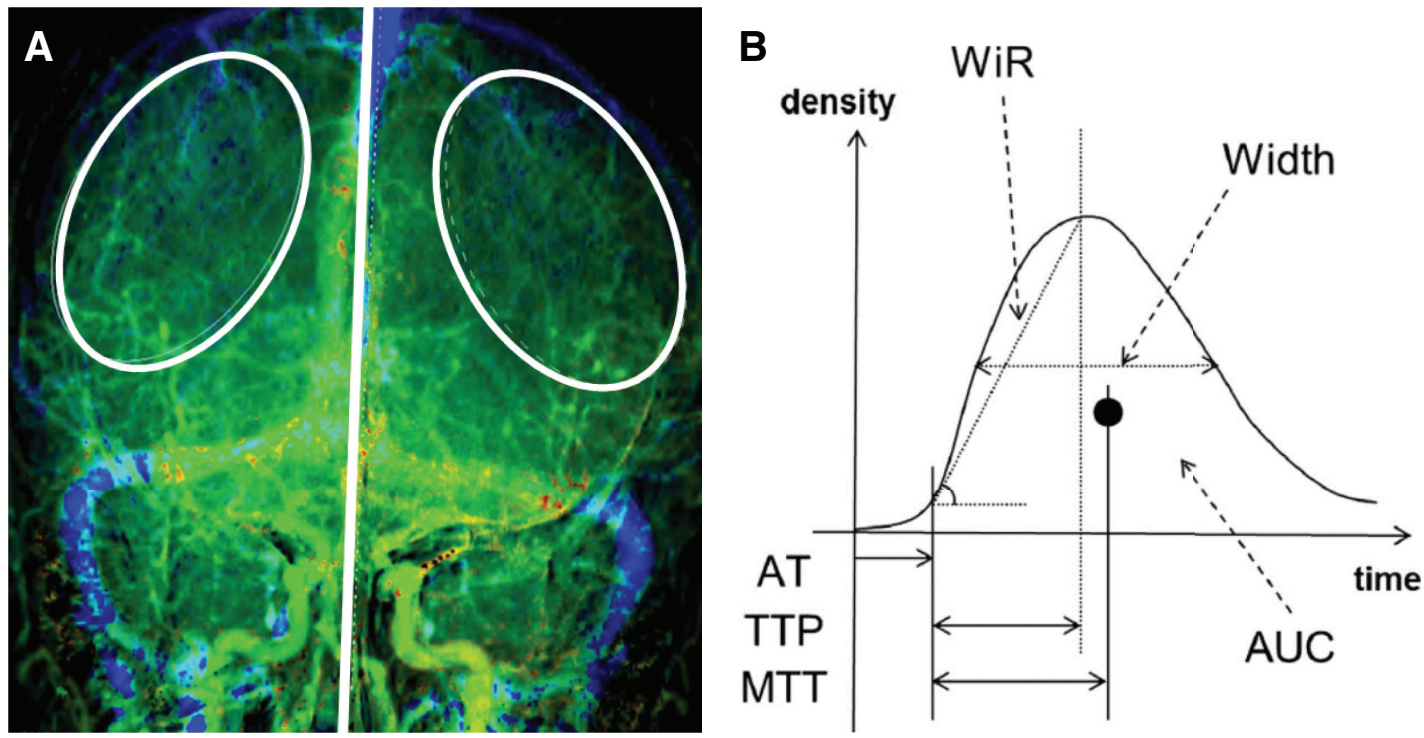

Fig. 1 (A) A 2D-perfusion image. A median line was established and the symmetric, oval regions of interest were established in the bilateral MCA regions. (B) Parameters obtained from the time-concentration curve. The black point indicates the intermediate point of the curve. AT of contrast medium, TTP, WiR, retention time of contrast medium (Width), AUC, and MTT. AT: arrival time; AUC: area under the curve; MCA: middle cerebral artery; MTT: mean transit time; TTP: time to peak; WiR: wash-in rate 
Table 1 Characteristics of all patients

\begin{tabular}{|c|c|c|c|c|c|c|c|c|c|c|c|c|}
\hline \multirow[b]{2}{*}{ Case } & \multirow[b]{2}{*}{$\begin{array}{l}\text { Age } \\
\text { (y.o.) }\end{array}$} & \multirow[b]{2}{*}{ Sex } & \multirow[b]{2}{*}{$\begin{array}{l}\text { Affected } \\
\text { side }\end{array}$} & \multirow{2}{*}{$\begin{array}{c}\text { Degree of } \\
\text { stenosis in } \\
\text { NASCET } \\
(\%)\end{array}$} & \multicolumn{6}{|c|}{ 2D-perfusion } & \multicolumn{2}{|c|}{ SPECT(\%) } \\
\hline & & & & & rTTP & rAT & rMTT & rAUC & rWiR & rWidth & $\begin{array}{c}\text { Stage II + I. } \\
\mathrm{R} \leq 30 \%\end{array}$ & $\begin{array}{c}\text { CVR in } \\
\text { whole } \\
\text { MCA area }\end{array}$ \\
\hline 1 & 69 & M & $\mathrm{R}$ & 76 & 1.07 & 1.03 & 1.07 & 1.46 & 1.23 & 1.16 & 3.7 & 17.0 \\
\hline 2 & 74 & M & $\mathrm{R}$ & 86 & 1.21 & 1.14 & 1.23 & 1.15 & 0.78 & 1.22 & 23.6 & 5.2 \\
\hline 3 & 70 & M & $\mathrm{R}$ & 95 & 1.43 & 1.25 & 1.48 & 1.11 & 0.52 & 1.53 & 57.1 & -3.7 \\
\hline 4 & 76 & M & $\mathrm{R}$ & 91 & 1.31 & 1.18 & 1.29 & 1.16 & 0.75 & 1.22 & 35.9 & -7.8 \\
\hline 5 & 76 & M & $\mathrm{R}$ & 70 & 1.13 & 0.94 & 1.09 & 1.32 & 1.16 & 1.04 & 14.8 & 30.8 \\
\hline 6 & 73 & M & $\mathrm{L}$ & 64 & 1.00 & 0.97 & 1.03 & 1.06 & 1.04 & 1.07 & 0.2 & 30.8 \\
\hline 7 & 56 & M & $\mathrm{L}$ & 55 & 1.08 & 0.94 & 1.04 & 0.88 & 0.81 & 1.00 & 1.0 & 58.1 \\
\hline 8 & 64 & M & $\mathrm{R}$ & 62 & 1.18 & 1.15 & 1.21 & 1.33 & 0.88 & 1.19 & 73.1 & -1.5 \\
\hline 9 & 75 & M & $\mathrm{R}$ & 70 & 1.06 & 1.18 & 1.03 & 0.86 & 0.82 & 0.98 & 1.8 & 39.2 \\
\hline 10 & 74 & $\mathrm{~F}$ & $\mathrm{R}$ & 53 & 1.03 & 1.08 & 1.03 & 1.11 & 1.04 & 1.02 & 0.0 & 91.2 \\
\hline 11 & 77 & $M$ & $\mathrm{R}$ & 54 & 1.00 & 1.07 & 1.00 & 1.09 & 1.00 & 1.16 & 1.6 & 36.9 \\
\hline 12 & 60 & $\mathrm{~F}$ & $\mathrm{R}$ & 76 & 1.10 & 1.16 & 1.09 & 1.20 & 0.98 & 1.05 & 0.6 & 56.5 \\
\hline 13 & 87 & $\mathrm{M}$ & $\mathrm{R}$ & 76 & 0.85 & 1.15 & 0.89 & 0.76 & 1.00 & 0.98 & 9.2 & -3.4 \\
\hline 14 & 77 & $M$ & $\mathrm{R}$ & 80 & 1.14 & 1.19 & 1.16 & 1.25 & 0.90 & 1.18 & 10.7 & 10.7 \\
\hline
\end{tabular}

AT: arrival time; AUC: area under the curve; CVR: cerebrovascular reserve; MCA: middle cerebral artery; MTT: mean transit time; NASCET: North American Symptomatic Carotid Endarterectomy Trial; SPECT: single photon emission computed tomography; TTP: time to peak; WiR: wash-in rate

was affected in 12 patients and the left side was affected in 2. According to the NASCET method, the mean percent stenosis was 71\% (53\%-91\%) (Table 1).

Spearman's correlation coefficient is shown in Table 2. There was a strong correlation between the rTTP on $2 \mathrm{D}$-perfusion and stage II + I.R of the $\leq 30 \%$ area on SPECT $(r=0.75, p=0.002)$. There was a strong correlation between the rMTT on 2D-perfusion and stage II + I.R of the $\leq 30 \%$ area on SPECT $(r=0.72, p=0.003)$ (Fig. 2). Furthermore, there was a positive correlation between the rWidth and stage II + I.R of the $\leq 30 \%$ area $(r=0.65$, $\mathrm{p}=0.012$ ). There was a negative correlation between the rWidth and CVR in the entire MCA area $(r=-0.67$, $\mathrm{p}=0.009)$.

\section{Case presentation}

Case No. 3 (Fig. 3)

\section{Patient: A 70-year-old man.}

Right cerebral infarction developed with a complaint of fugacious amaurosis of the right eye. Magnetic resonance angiography (MRA) and cervical ultrasonography revealed marked stenosis of the right cervical internal carotid artery. Treatment for acute ischemic stroke was prioritized. Cerebral angiography and ACZ-loaded SPECT with ${ }^{1,2,3}$ I-IMP were performed 3 months after onset.

Pseudo-occlusion of the right cervical internal carotid artery was observed (Fig. 2A). Furthermore, 2D-perfusion confirmed a difference in the time-concentration curve between the bilateral hemispheres (Fig. 2B). The rMTT, rTTP, and rWidth were $1.48,1.43$, and 1.53 , respectively.

On SEE analysis, the stage II + I.R of the $<-30 \%$ area accounted for $57.1 \%$ of the affected-side MCA area and the CVR in the entire MCA area was $-3.7 \%$, being markedly reduced (Fig. 2C).

Table 2 Spearman correlation coefficient between the relative ( $r$ ) parameters of 2D-perfusion and SPECT

\begin{tabular}{cccccrrrrr} 
& \multicolumn{4}{c}{ 2D-perfusion } \\
\cline { 3 - 7 } & & rTTP & rAT & rMTT & rAUC & rWiR & rWidth \\
SPECT & Stage II + I.R $<-30 \%$ & $0.75^{*}$ & 0.43 & $0.72^{*}$ & 0.41 & -0.5 & $0.65^{*}$ \\
& CVR in whole MCA area & $-0.54^{*}$ & 0.44 & $-0.57^{*}$ & -0.31 & 0.38 & $-0.67^{*}$ \\
\hline
\end{tabular}

*Statistically significant.

AT: arrival time; AUC: area under the curve; CVR: cerebrovascular reserve; I R: increase ratio; MCA: middle cerebral artery; MTT: mean transit time;

SPECT: single photon emission computed tomography; TTP: time to peak; WiR: wash-in rate 

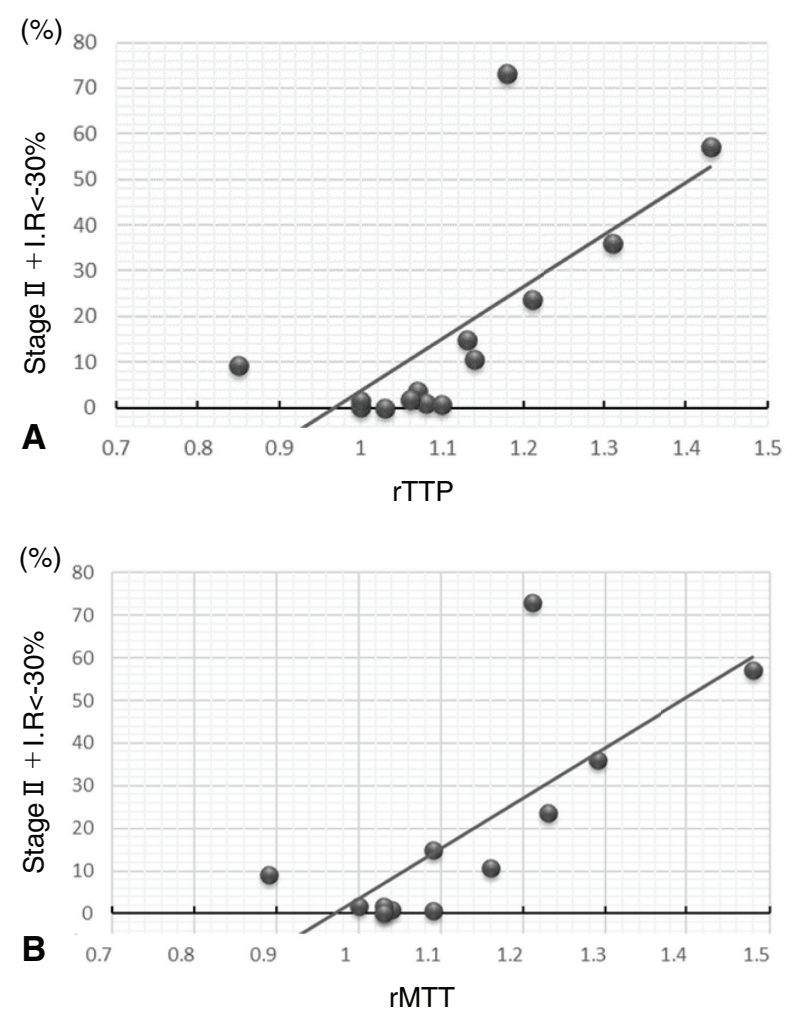

Fig. 2 (A) Scatter diagram and approximation straight line of the rTTP and stage II + I.R of the $\leq 30 \%$ area. (B) Scatter diagram and approximation straight line of the rMTT and stage II + I.R of the $\leq 30 \%$ area. I.R: increase ratio; MTT: mean transit time; TTP: time to peak

\section{Discussion}

In this study, the rTTP and rMTT on 2D-perfusion were strongly correlated with the degree of the stage II + I.R of the $\leq 30 \%$ area, with a marked reduction in the CVR on SEE analysis based on ACZ-loaded SPECT with ${ }^{1,2,3}$ I-IMP in patients with unilateral cervical internal carotid artery stenosis. No study has examined the correlation between 2D-perfusion parameters and a reduction in CVR on SPECT. 2D-perfusion may replace conventional examinations for CVR assessment.

In this study, a receiver operating characteristic (ROC) curve for extracting patients in whom the stage II + I.R of the $<-30 \%$ area accounted for $>9 \%$, especially those with an extensive CVR-reduced area, was prepared, and the cutoff values of rTTP and rMTT were 1.13 and 1.09 , respectively. The sensitivity and specificity of the former were 1.00 and 0.857 , respectively. Those of the latter were both 0.857 . These parameters may become reference values to be clinically applied (Fig. 4). The TTP and MTT depend on the time of brain tissue perfusion of contrast medium; CVR is not directly evaluated. However, Asai et al. ${ }^{11)}$ established the region of interest in the bilateral hemispheres on 2D-perfusion in a balloon obliteration study, and reported that the TTP and MTT on the occluded side
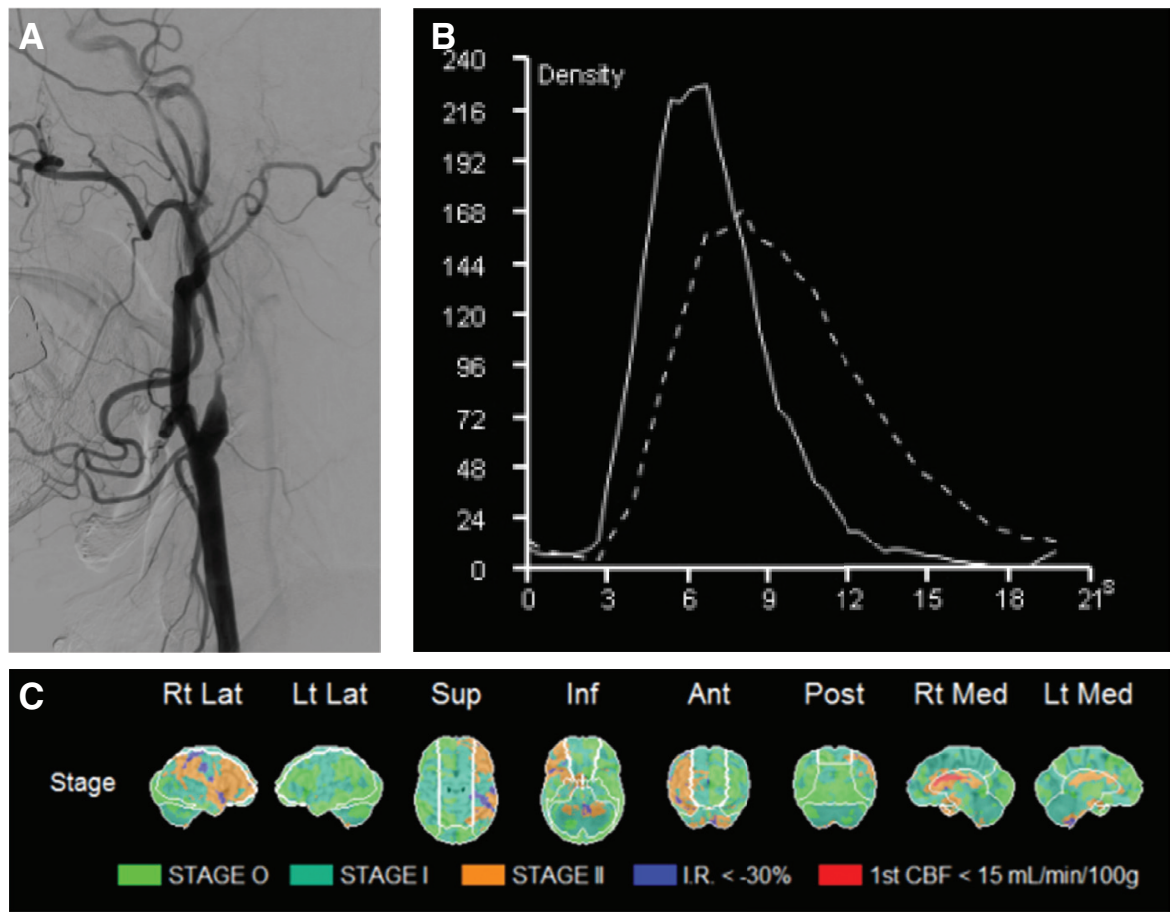

Fig. 3 A representative case (No. 3 in Table 1). (A) Lateral view on right common carotid angiography. Marked stenosis was observed at the origin of the internal carotid artery. Collapse of the peripheral internal carotid artery was noted. According to the NASCET method, the percent stenosis was $95 \%$. (B) A time-concentration curve in the region of interest on 2D-perfusion imaging. Solid line: Unaffected side (left), Dotted line: Affected side (right). (C) SEE analysis. On the right side, the stage II + increase ratio of the $\leq 30 \%$ area was extensive. NASCET: North American Symptomatic Carotid Endarterectomy Trial; SEE: Stereotactic extraction estimation 

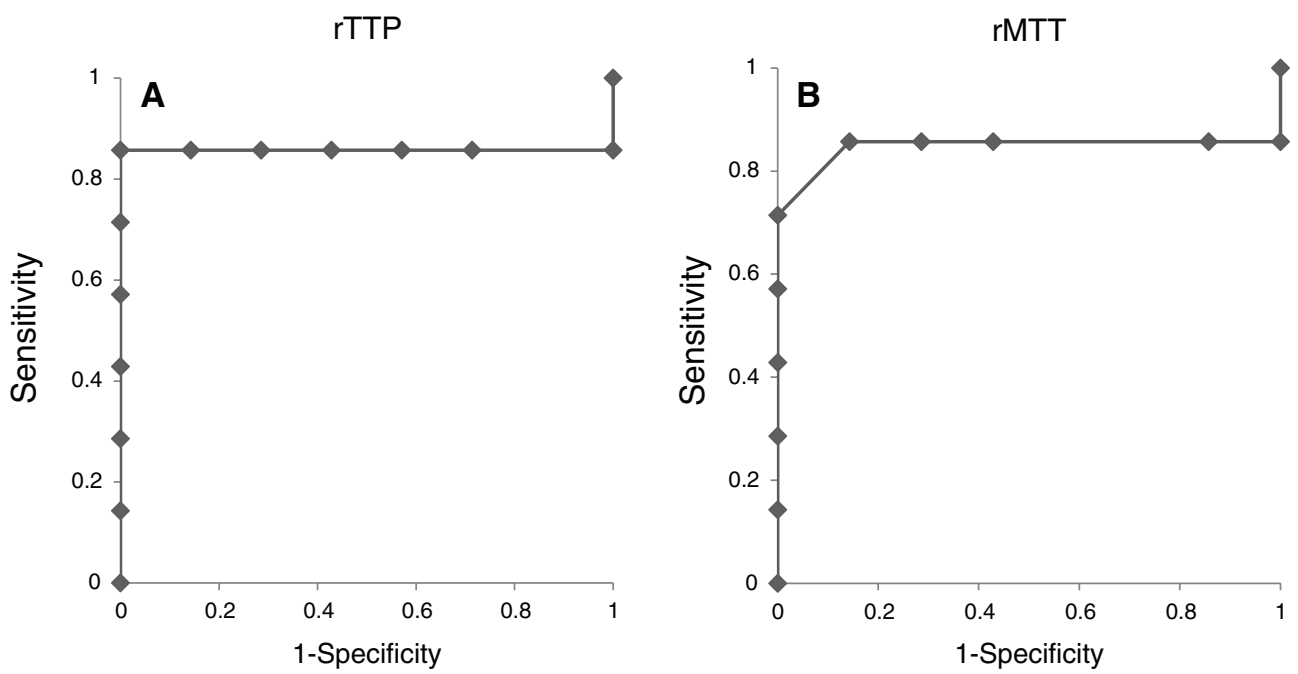

Fig. 4 ROC curves to extract patients with a stage II + I.R of the $\leq 30 \%$ area accounting for $>9 \%$. (A) ROC curve for rTTP. (B) ROC curve for rMTT. MTT: mean transit time; ROC: receiver operating characteristic; TTP: time to peak

were significantly longer than those on the opposite side in the non-resistance group. These parameters may predict a CVR-decrease-related reduction in ischemic tolerance.

Regarding the other parameters of 2D-perfusion, the Width, which depends on the interval from contrast medium arrival, as described for the TTP and MTT, was correlated with SPECT. The AT refers to the time of contrast medium arrival, but this may depend on the anatomical vascular length; there was no correlation with SPECT. Furthermore, neither the WiR nor AUC, which depend on the concentration, was correlated with SPECT. This was possibly because the contrast medium discharged in the ascending aorta was influenced by the cardiac output, making the intensity of contrast medium irregular. In the future, a review in consideration of heart function should be conducted.

The degree of intracranial collateral pathway development cannot be considered by evaluating the grade of cervical internal carotid artery stenosis alone; there is often dissociation from the actual state of cerebral blood flow. In the method used in this study, perfusion of the contrast medium discharged in the ascending aorta was evaluated in the region of interest; therefore, the blood flow of all intracranial blood vessels was included, facilitating the assessment of the degree of collateral pathway development.

In patients with internal carotid artery stenosis, a marked reduction in CVR on PET or SPECT is a risk factor for ischemic stroke. ${ }^{5,6}$ Furthermore, a reduction in the responsiveness to ACZ loading is a predictor of hyperperfusion after carotid endarterectomy or carotid artery stenting. ${ }^{1-4)}$ Therefore, these examinations are routinely performed for condition assessment and preoperative detailed examination. However, institutions where PET can be conducted are limited and $\mathrm{ACZ}$ was reported to induce serious adverse reactions. ${ }^{7,8)}$ If $2 \mathrm{D}$-perfusion can replace these examinations for CVR assessment, they may be omitted at institutions where PET cannot be conducted or in high-risk patients for adverse reactions to ACZ.

2D-perfusion is a cerebral-blood-flow-evaluating method that can be carried out during angiography. In addition to morphological assessment by angiography, functional assessment can be simultaneously performed without transferring patients. Real-time CVR assessment may facilitate determining the indication of treatment/timing for atherosclerotic stenotic lesions detected after thrombectomy or arranging strict strategies through preoperative evaluation of the risk of hyperperfusion after carotid artery stenting.

However, this study has several limitations. First, only unilateral lesions can be evaluated using this analytical method. The method to calculate the affected-to-unaffected side ratio is advantageous in that it excludes many confounding factors related to absolute-value assessment. However, internal carotid artery stenosis, as an arteriosclerotic lesion, is often complicated by a contralateral stenotic lesion; the analytical method adopted in this study cannot be used in some cases. Second, 2D-perfusion images may be influenced by body motion. Slight movement during 
imaging markedly reduces the accuracy of analysis. Therefore, it is important to sufficiently explain the possibility of discomfort at the center of the chest due to the infusion of high-dose contrast medium immediately before 2D-perfusion imaging, and to tell the patient not to swallow saliva or cough. Furthermore, imaging is impossible for some patients in whom the dose of contrast medium is limited due to renal dysfunction. However, in our patients, the dose of contrast medium was reduced in the presence of mild renal dysfunction and images sufficient for analysis were obtained on 2D-perfusion imaging with the contrast medium diluted at a ratio of 1.5 .

\section{Conclusion}

In patients with unilateral cervical internal carotid artery stenosis, 2D-perfusion analysis may facilitate the prediction of the degree of a markedly circulatory-reservereduced area.

\section{Disclosure Statement}

The authors declare no conflict of interest.

\section{References}

1) Misaki K, Uchiyama N, Inaki A, et al: Objective evaluation of cerebrovascular reactivity for acetazolamide predicts cerebral hyperperfusion after carotid artery stenting: comparison with region of interest methods. $J$ Neuroradiol 2018; 45: 362-367.

2) Hosoda K, Kawaguchi T, Ishii K, et al: Prediction of hyperperfusion after carotid endarterectomy by brain SPECT analysis with semiquantitative statistical mapping method. Stroke 2003; 34: 1187-1193.

3) Komoribayashi N, Ogasawara K, Kobayashi M, et al: Cerebral hyperperfusion after carotid endarterectomy is associated with preoperative hemodynamic impairment and intraoperative cerebral ischemia. J Cereb Blood Flow Metab 2006; 26: 878-884.

4) Ogasawara K, Sakai N, Kuroiwa T, et al. Intracranial hemorrhage associated with cerebral hyperperfusion syndrome following carotid endarterectomy and carotid artery stenting: retrospective review of 4494 patients. J Neurosurg 2007; 107: 1130-1136.

5) Morales-Valero SF, Lanzino G. Asymptomatic carotid artery stenosis: time to rethink our therapeutic options? Neurosurg Focus 2014; 36: E2.

6) Gupta A, Chazen JL, Hartman M, et al: Cerebrovascular reserve and stroke risk in patients with carotid stenosis or occlusion: a systematic review and meta-analysis. Stroke 2012; 43: 2884-2891.

7) Zimmermann $S$, Achenbach S, Wolf M, et al: Recurrent shock and pulmonary edema due to acetazolamide medication after cataract surgery. Heart Lung 2014; 43: 124-126.

8) Vogiatzis I, Koulouris E, Sidiropoulos A, et al: Acute pulmonary edema after a single oral dose of acetazolamide. Hippokratia 2013; 17: 177-179.

9) Ogasawara K. Brain perfusion measurement for patients undergoing reconstructive surgery for main cerebral arteries in the era of restriction for acetazolamide challenge. Jpn J Neurosurg (Tokyo) 2016; 25: 834-843 (in Japanese).

10) Asai $\mathrm{K}$, Imamura $\mathrm{H}$, Mineharu $\mathrm{Y}$, et al. X-ray angiography perfusion analysis for the balloon occlusion test of the internal carotid artery. J Stroke Cerebrovasc Dis 2015; 24: 1506-1512.

11) Asai $K$, Nakamura $H$, Watabe $T$, et al: $X$-ray angiography perfusion imaging with an intra-arterial injection: comparative study with 150 -gas/water positron emission tomography. J Neurointerv Surg 2018; 10: 780-783.

12) Tani $S$, Imamura $H$, Asai $K$, et al. Comparison of practical methods in clinical sites for estimating cerebral blood flow during balloon test occlusion. J Neurosurg 2018; 131: 1-7.

13) Mizumura S, Nakagawara J, Takahashi M, et al: Threedimensional display in staging hemodynamic brain ischemia for JET study: objective evaluation using SEE analysis and 3D-SSP display. Ann Nucl Med 2004; 18: 13-21. 